\title{
Deformation measurement within adhesive bonds of aluminium and CFRP using advanced fibre optic sensors
}

\author{
Hinrich Grefe*, Dennis Weiser, Maja Wanda Kandula, and Klaus Dilger \\ Institute of Joining and Welding, Technische Universitaet Braunschweig, Braunschweig, Germany
}

Received: 28 November 2019 / Accepted: 19 February 2020

\begin{abstract}
Monitoring the deformation within an adhesive joint during the curing cycle provides valuable information regarding the build-up of thermal strain and stress. Distributed fibre optic sensors are very useful for spatial continuous measurements of deformation or temperature. Integrated into a hybrid joint, the thermal curing process of the adhesive can be monitored. This detailed insight into the joint helps to understand the deformation and thereby also the resulting stress. Analysing the deformation process establishes the foundation to adapt techniques to reduce the thermally induced deformation and thereby the resulting stress.
\end{abstract}

Keywords: Composites / curing / deformation / CTE-mismatch

\section{Introduction}

Fibre reinforced plastics gain importance as lightweight material. They are commonly combined with a variety of other materials. Due to the diverse material properties an appropriate joining technique and joint design needs to be adapted for each individual material combination. One of the main challenges is their difference in thermal expansion coefficients which causes deformation and stress in the components or assemblies.

\subsection{Thermal induced stresses and deformation in hybrid joints}

Hybrid joints or hybrid constructions for structural components often utilise adhesive bonding as a joining technique. In high volume productions, such as the automotive industry, the adhesive is often cured during a high temperature process such as the paint dryer oven. The cured part might also be subjected to other high temperature processes in following production stages. Hence either the thermal expansion is fixed in place during the curing causing stress and deformation at ambient conditions or the already cured joint is subjected to stress and deformation during the following thermal processes [1]. An analysis of the involved materials and processes is therefore essential to understand their interaction. This also includes the properties of the adhesives and their behaviour under mechanical loading or deformation while

\footnotetext{
* e-mail: h.grefe@tu-braunschweig.de
}

the adhesive is in different stages of curing and under different temperatures [2]. After understanding the behaviour of the individual components, consequently the analysis of the joint or the complete assembly is the next step. This provides more realistic and therefore more truthful testing conditions, but also the challenge to observe and measure larger and more complex specimen.

Digital image correlation (DIC) is a good example of state-of-the-art technology being used in research applications. However, this technique only works on visible surfaces. Due to the opaque nature of most structural components it is not possible to analyse the condition and behaviour in a joint using DIC. Tactile sensors such as strain gauges on the other hand are well suited to be imbedded within a joint. Strain gauges generally operate as one singular measurement spot rendering them unsuited for a spatially continuous measurement.

\subsection{Using optical fibres as sensors}

The most significant advantage of measuring strain with optical fibres is the maximal attainable spatial resolution.

Conventional strain measuring techniques such as strain gauges operate as isolated point-by-point sensors, therefore they are only capable to monitor strain or deformation in one localized spot. To obtain a measurement with spatial distribution, multiple sensors have to be used and individually installed for each data point as shown by da Silva et al. [3] using three individual sensors to measure the strain distribution at three separate points within a single lap shear joint. The spatial resolution of the measurement is limited by the geometrical size of the 
sensors. This applies to resistive strain gauges as well as to localized optical strain gauges such as fibre Bragg grating sensors (FBG).

Using optical fibres to measure strain, a general differentiation has to be made between spot measurements and distributed, continuous measurements. Generally both types operate using optical fibres, detecting the strain of the fibre using light. Fibre Bragg grated sensors have a punctual sensor spot which is formed by an optical grate embedded into the sensor. A deformation of the sensor fibre therefore results in a change of the grate spacing and causes a change in the reflected wavelength. While it is possible to place multiple grates with different wavelength within one fibre (multiplexed FBG), the operating principle as series of punctual sensor remains the same. The resolution achievable with multiplexed FBG or other multiple sensors is restricted to the geometric properties of the fibre or the individual sensor. This would still allow for blind spots where a potential strain build-up would not be detected [4]. Distributed fibre optic sensors (FOS) provide a spatially continuous measurement, eliminating blind spots caused by sensor spacing. A general overview of the different available FOS for distributed or spot measurement and their appliance is given by Ramakrishnan et al. [5].

Continuous or distributed fibre sensors operate based on the properties or characteristics of the fibre itself and analyse the light scattered and reflected by the fibre in response to an incoming pulse. A general distinction can be made between sensor systems working either with elastically reflected light, the Rayleigh scattering, or with inelastic Brillouin or Raman scattering. Brillouin scattering describes an inelastic interaction between optical photons and acoustic phonons, utilising this effect temperature differences and strain can be measured. Sensing ranges up to $100 \mathrm{~km}$ are possible without the need of in-line amplifiers with a typical spatial resolution of $2 \mathrm{~m}$. Special "pre-pumping" techniques were shown to improve the resolution to $2 \mathrm{~cm}$ within a $2 \mathrm{~km}$ sensing length [6]. The Raman scattering is an inelastic exchange of energy between a photon and solid matter which can be utilised to detect changes in temperature within an optical fibre. Raman sensors are mainly used as temperature monitoring devices in fixed structures such as tunnels $[7,8]$ and is able to detect temperature variations as small as $0.1^{\circ} \mathrm{C}[5]$. Rayleigh scattering describes an elastic effect, in optical fibres it can be caused by variations in density or the refractive index. These variations are characteristic for the individual fibre and its condition. A measurement taken at a known condition of the fibre can be used as reference to determine any occurring change $[9,10]$. Any other taken measurement can then be compared to the reference and interpreted as mechanical strain as well as thermal deformation. To obtain a measurement, two methods can be used: the optical frequency domain reflectometry (OFDR) or the optical time domain reflectometry (OTDR). The OTDR is based on the time of flight, measuring changes in the distance between the characteristic scatter patterns. Depending on the principles used, the sensor spacing varies between $0.5 \mathrm{~m}$ and $1 \mathrm{~m}$ [11]. The OFDR detects the changes in frequency or rather the wavelength of the Rayleigh scattering. The wavelength of the scattering, although randomly distributed, is a unique and fixed property of the given fibre [12]. The principle procedure is similar to the use of FBG, a change in the reflected wavelength represents a change in the properties of the fibre: a deformation. A tuneable laser generates the variable wavelength, the fibres reflections create an interferences in the Mach-Zehnder interferometer which is then analysed [13]. The limiting factor using OFDR is the coherence length of the tuneable laser source [14].

\subsection{Strain measurement using optical fibres}

The small diameter of the fibres used as FOS is one of their main advantages. Including the polyimide coating most fibres' thickness is between $155 \mu \mathrm{m}$ and $250 \mu \mathrm{m}$. In composite applications, the fibre can therefore be directly integrated into the composite structure during manufacturing. It is shown in Jothibasu et al. [4] that with sufficient thickness of the core, the sensor fibre is not influenced by slight deformations of the coating which might occur during the manufacturing process. Furthermore, the seamless integration of the sensor does not interfere with the behaviour of the composite. The measurements taken by the imbedded fibre were compared to a surface bonded electrical resistance strain gauge and confirmed. Rahim et al. [15] performed a direct comparison between an electrical foil strain gauge and a FOS. Both sensors were placed on the surface of a fibreglass specimen which was subjected to periodic four point bending. For low strain levels $(2000 \mu \mathrm{m} / \mathrm{m}$ or $0.2 \%)$ both sensors performed within their specifications, no sign of signal drift was recorded. Raising the strain level to $4000 \mu \mathrm{m} / \mathrm{m}$ or $0.4 \%$ resulted in a severe drift of the electrical strain gauge which built up to $45 \%$ deviation after 1000 cycles. During the further measurement, the FOS did not show any sign of drift up to the end of the experiment at 27000 cycles. Wong et al. [16] investigated the strain at the surface of an composite step lap joint during fatigue testing using a FOS. They revealed an unexpected, non-uniform strain distribution along the joint. A measurement by singular sensors would have missed this phenomenon even using as many sensors as geometrically possible. The continuous measurement along the fibre is especially useful to detect singularities such as cracks within a concrete beam $[17,18]$. In Chen et al. [19] a carbon fibre reinforced polymer (CFRP) strip was used to strengthen a concrete beam, FOS were applied to the steel rebar inside the concrete and on the CFRP surface, adhesively bonded to the concrete beam. This setup was used to detect cracks in the concrete and correlate them with increasing strain within the externally bonded CFRP strip. Also the progressing failure within the adhesive and the debonding were monitored. The deformation during the curing process and the resulting properties of adhesively bonded CFRP-steel joints were investigated with FOS by Dietrich [20]. Using a setup with a fixed bearing on one side and a floating bearing on the other it was determined that a change in the fibre architecture of the CFRP has the utmost impact to the strain distribution. Using a CFRP with no fibres in the $0^{\circ}$ orientation (main direction of thermal expansion) the 


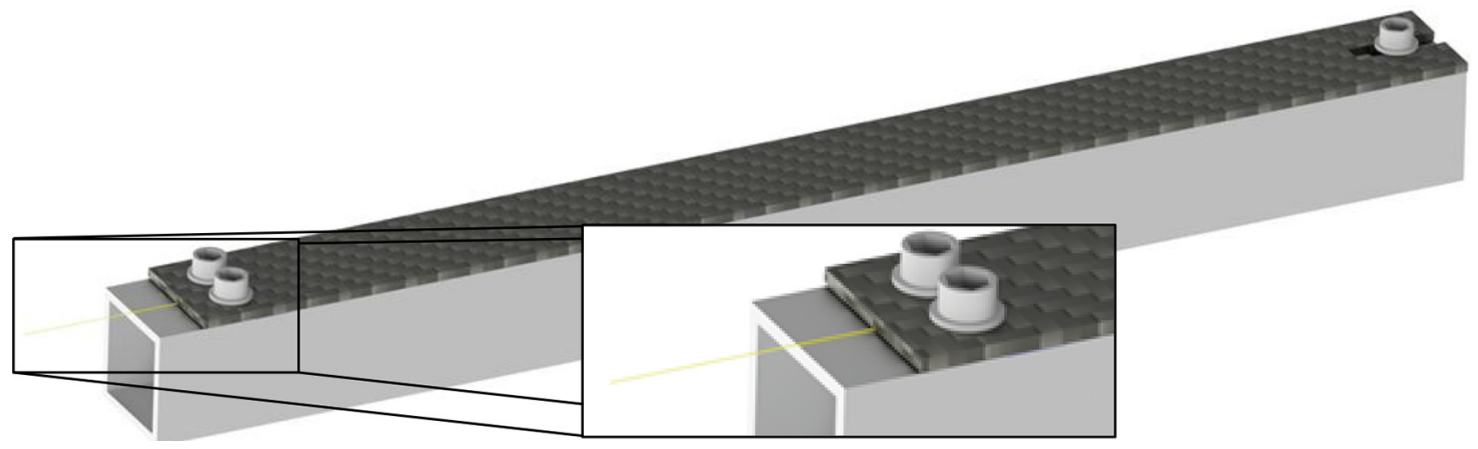

Fig. 1. CAD model of the fixed bearing specimen.

residual deformation caused by the high temperature curing cycle was mostly located within the composite material. With other laminates the $0^{\circ}$ oriented fibres restricted deformation within the composite. This resulted in strain in the adhesive layer as well as in the steel. With an adhesive thickness of $0.3 \mathrm{~mm}$, a curing temperature of $180^{\circ} \mathrm{C}$ and a specimen length of $450 \mathrm{~mm}$ adhesive failure occurred and destroyed the sensor in the adhesive layer. Therefore the further testing was performed with $1.5 \mathrm{~mm}$ and $3.0 \mathrm{~mm}$ adhesive thickness to avoid loss of data caused by broken sensors.

\section{Experimental design}

\subsection{Materials}

Exploring the potential of FOS to monitor the deformation in adhesively bonded hybrid joints, a material combination of unidirectional CFRP and aluminium square tube was chosen. Both materials are commonly used in lightweight applications and have different thermal expansion coefficients. Therefore large deformations caused by thermal expansion can be expected. The CFRP specimens were fabricated as $3 \mathrm{~mm}$ thick sheets using HexPly 6376 in the recommended autoclave process $\left(2 \mathrm{~h} @ 175^{\circ} \mathrm{C}\right)$. The aluminium square tubes dimensions are $25 \times 25 \mathrm{~mm}$ with a wall thickness of $1.5 \mathrm{~mm}$ extruded from AlMgSi1. SikaPower-477 R was used as adhesive, already containing glass beads with $300 \mu \mathrm{m}$ diameter to set the adhesive thickness to $0.3 \mathrm{~mm}$. To monitor the deformation, a Luna ODiSI-B interrogator was used with a single mode $155 \mu \mathrm{m}$ diameter fibre.

All bonding surfaces were prepared by cleaning with isopropyl alcohol, grit blasting using aluminium oxide (for CFRP the pressure was reduced to avoid damage within the laminate) and cleaning with isopropyl alcohol afterwards. No adhesion promoters, boosters or primers were used.

\subsection{Specimen design}

With the general materials for the test defined, the first tests were performed using a combination of fixed and floating bearing to maximize the thermal deformation. The floating bearing was provided by a slot in the CFRP and a slightly tightened M5 screw to prevent sliding to the side. Two M5 screws were set tight for the fixed bearing on the opposite side distance pieces were used to protect the optical fibre from being crushed during fastening (Fig. 1). The adhesive was applied and spread over the specimen width. The optical fibre was embedded within the adhesive and the surface was smoothened afterwards. The CFRP was added and excess adhesive was squeezed out and removed.

The assembly was then set to cure at $120^{\circ} \mathrm{C}$ for $45 \mathrm{~min}$. During the heating cycle a very slight, homogeneous deformation was measured, caused by the shear between aluminium and CFRP. The measurement taken during the cooling phase was rather erratic as shown in Figure 2, the zero reference was set to the aluminium edge on the fixed bearing side. The deformation within the adhesive was expected to be at the highest at the floating bearing with a decrease towards the fixed bearing. The fixed bearing was designed to prohibit all relative movement and thereby avoid any shear or deformation.

The sensor with its $155 \mu \mathrm{m}$ diameter was not fixed in position and therefore able to move within the $300 \mu \mathrm{m}$ adhesive layer during the heating and curing of the specimen. This allowed an undulation of the sensor between the adherents, which was also observed by da Silva et al. reviewing the use of FBG in single lap shear tests [3]. The results of the measurement during the cooling process can be explained respectively: Compression was detected where the fibre was closer to the aluminium, elongation in areas close to the CFRP. The acquired measurement is therefore not suited to extract conclusive information. The sensor has to be mounted in a predefined position to determine from which part of the joint the measurement was taken. Reducing the undulation will decreases inaccuracies caused by the angle between the fibre and the main expansion direction. To embed the sensor into the aluminium tube a $0.1 \mathrm{~mm}$ deep and approximately $0.2 \mathrm{~mm}$ wide slot was machined into the bonding surface and the sensor was set in using high temperature cyanoacrylate (WIKO SG HT 2500). A cross section of the final specimen with the embedded fibre is shown in Figure 3.

As second observation, the measured strain is approximately similar on both ends; therefore, the fixed bearing must be insufficient. Different approaches were made to improve the bearing but ultimately it was not possible 


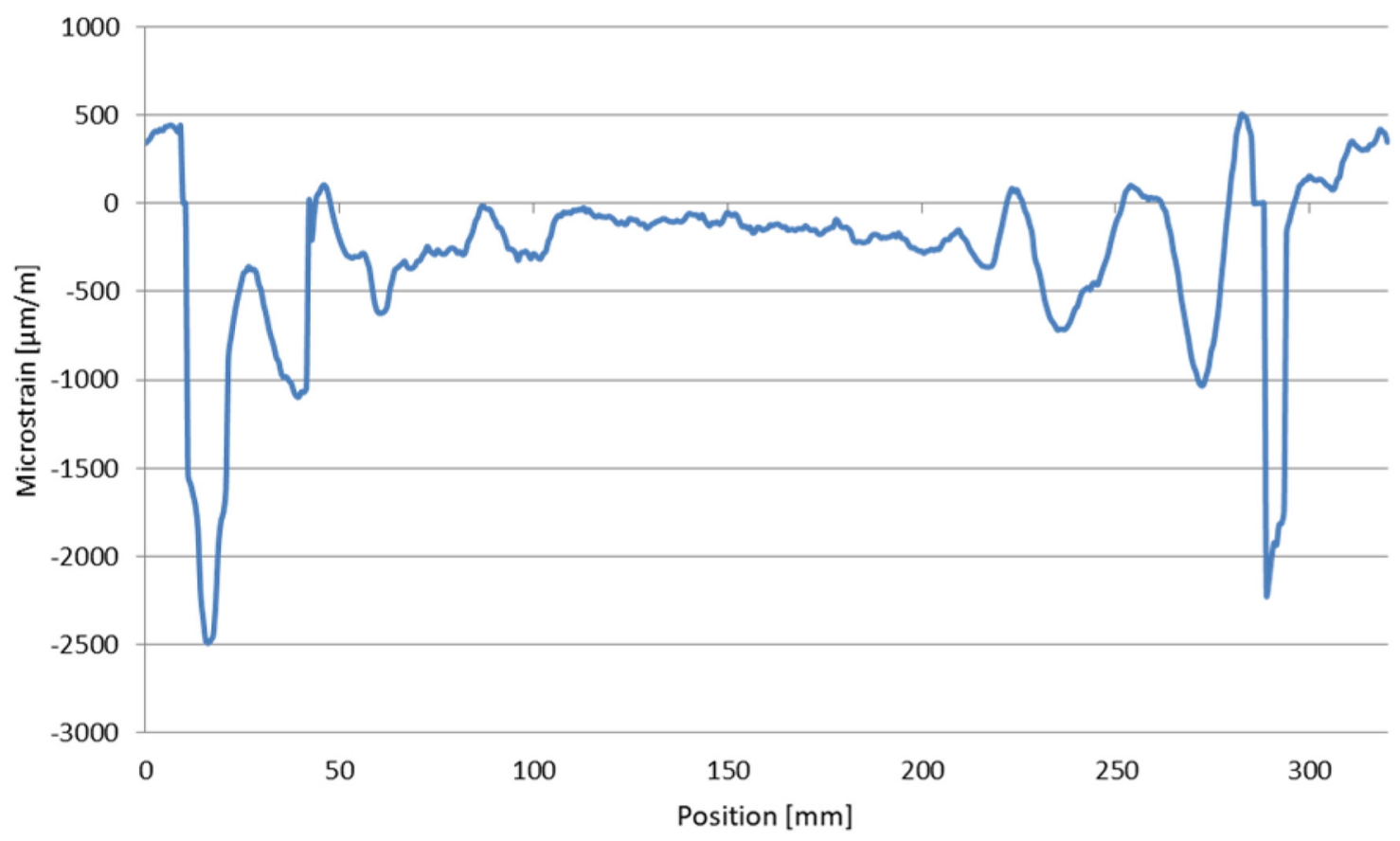

Fig. 2. Deformation after curing of the fixed bearing specimen.

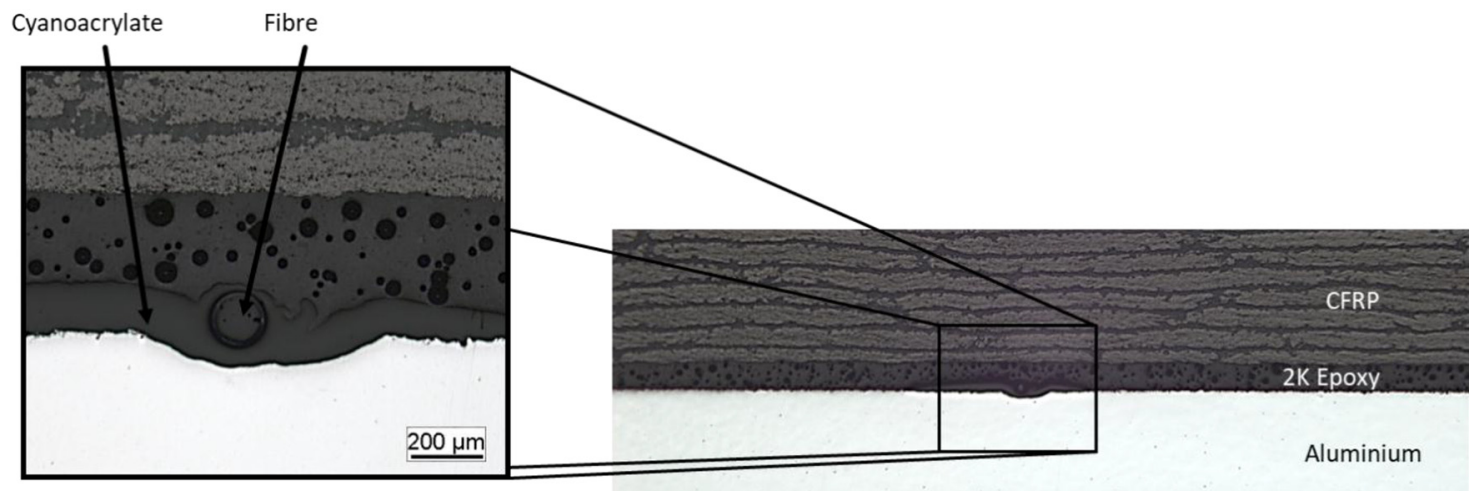

Fig. 3. Cross section of the final specimen design.

(with acceptable effort) to create a fixed bearing which did neither generate deformation on its own nor allow deformation to build up during the curing cycle. The specimen therefore was redesigned without bearings and increased in length to achieve a realistic behaviour. An additional free length of aluminium was added on either side to improve the measurement of the stress concentration towards the edges of the joint and avoid any interference that might be caused within the first millimetres of the embedded fibre.

\subsection{Test setup and measurement}

For the final design, the length of the aluminium tube was set to $420 \mathrm{~mm}$, the CFRP was cut to $350 \mathrm{~mm}$. After milling the groove and embedding the sensor, a preliminary measurement at a temperature of $130^{\circ} \mathrm{C}$ was performed to ensure a sufficient bond between fibre and aluminium. The structural adhesive was then directly applied to the aluminium using a static mixer and spread evenly; afterwards the CFRP was placed on top. Excess adhesive was squeezed out and removed. The assembled specimen was set in a support frame and held down by eight spring loaded plungers, each applied approximately $5 \mathrm{~N}$ (Fig. 4). A preheated convection oven was used to cure the adhesive at $125^{\circ} \mathrm{C}$ for $45 \mathrm{~min}$. The cool down afterwards was recorded as well.

\section{Results}

All recorded data has been referenced to a measurement taken after the preliminary heating and before applying the structural adhesive. This measurement was taken at an 


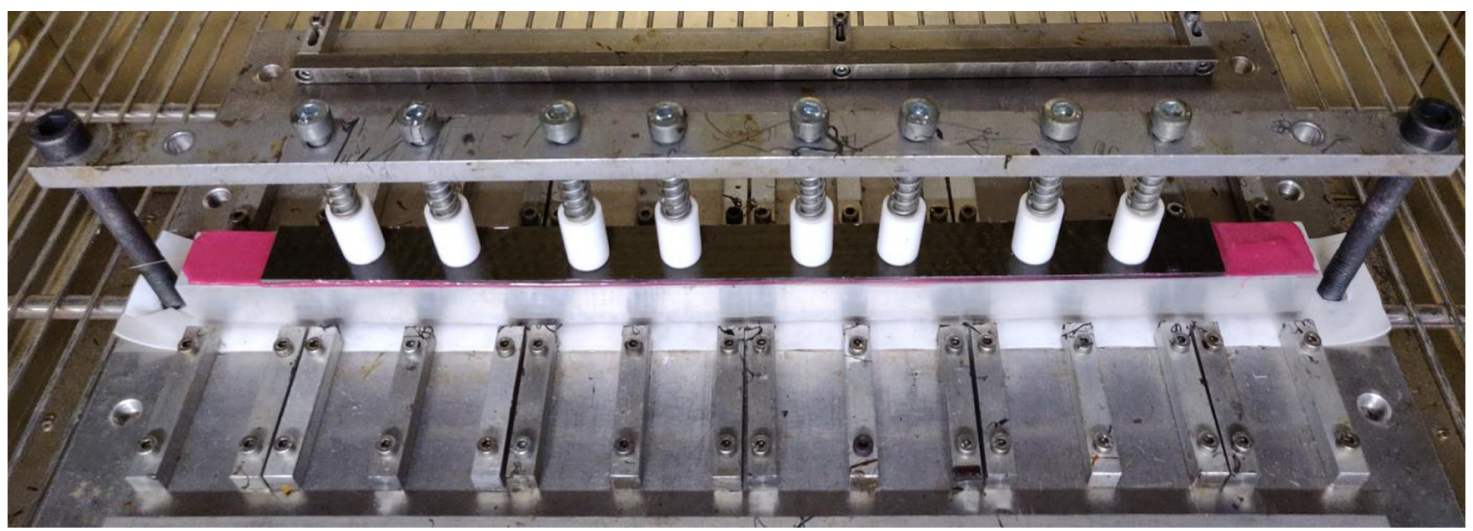

Fig. 4. Specimen and fixture in oven.

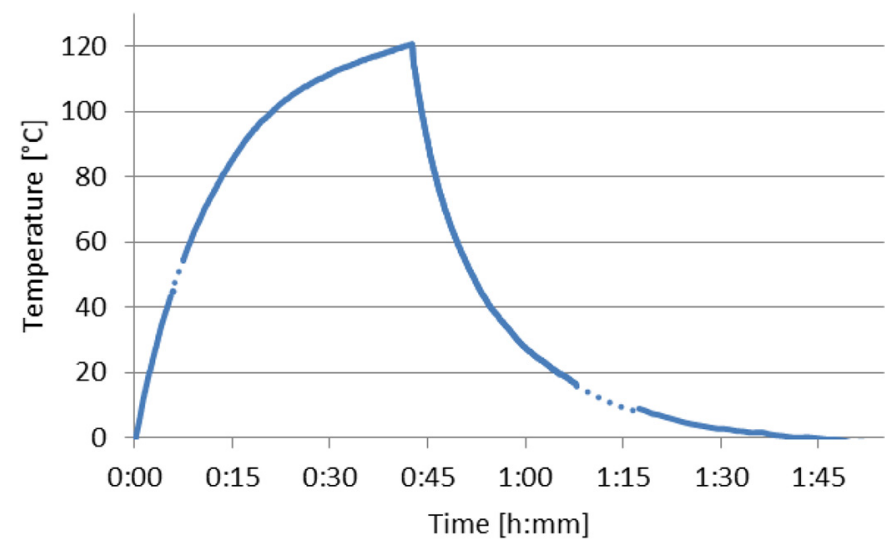

Fig. 5. Temperature curve measured by thermal expansion.

ambient temperature of $18^{\circ} \mathrm{C}$ represents the baseline for any deformation. To smooth the data, a rolling average was formed with a width of $1.94 \mathrm{~mm}$ and a uniformly balanced rolling average for the duration of 5 seconds. Instead of a direct temperature measurement by a thermocouple, the embedded fibre measured the deformation from the parts of the aluminium which were not used to form the joint (Fig. 5). The final temperature of $120^{\circ} \mathrm{C}$ was confirmed using a Type-K thermocouple. Due to the very good thermal conductivity of the material, the large surface area of the aluminium tube compared to its mass and the observed behaviour during the preliminary heating as well as the final test, the temperature within the aluminium is considered homogenous. The gaps (dotted line) in the graph result from buffer overflows during the data acquisition.

The rise in temperature causes thermal expansion and therefore a uniform deformation within the aluminium. After 18 minutes the measured strain in the centre of the joint (position 1142-1167 mm) increases significantly slower than at the free ends (position 950-975 mm). This is caused by the adhesive which forms the first crosslinks, obstructing the adherents thermal expansion. The observed behaviour is an indication for the passing of the gel-point. Figure 6 visualizes the diverging deformation over time for both indicated areas. The deformation

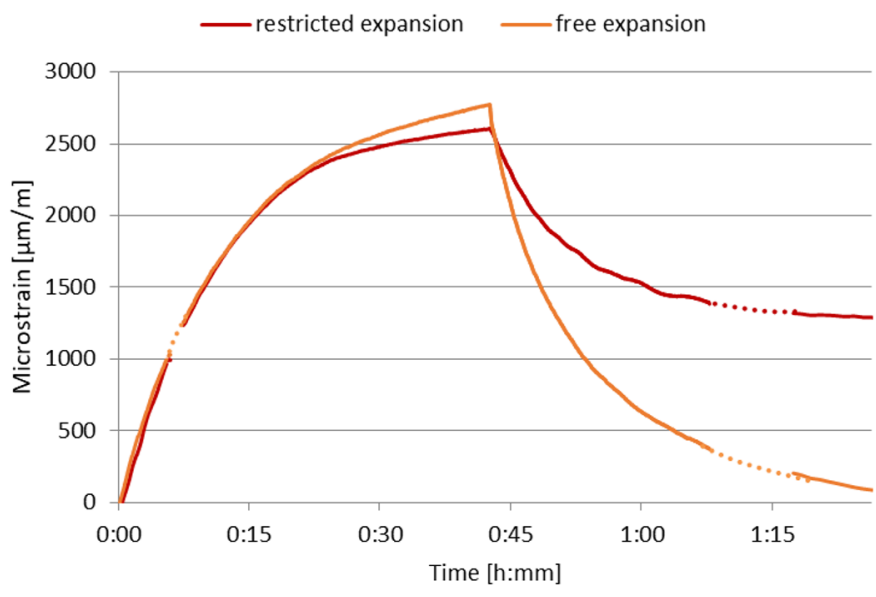

Fig. 6. Comparison between free expansion and restricted expansion.

gradient over the whole specimen length can be seen in Figure 7 for the characteristic timestamps; the dashed lines indicate the dimensions of the CFRP as reference.

After 45 minutes of curing, the specimen was placed at an ambient temperature of $18^{\circ} \mathrm{C}$ to cooldown. This progress can also be seen in the deformation over time visualisation in Figure 6. The formerly thermal expansion is now fixed in place by the adhesive. During the cool down the aluminium tube contracts as far as the elastic deformation in the rest of the joint allows it. This results in shear within the adhesive, bending of the specimen and compression of the CFRP. Figure 8 shows the measured deformation within the aluminium. The bending and deformation actually causes compression stress within the tube as can be seen at positions $979 \mathrm{~mm}$ and $1330 \mathrm{~mm}$ which also mark the edges of the CFRP. In the centre of the joint, the remaining deformation has a nearly constant plateau. This linear area indicates that nearly no loads are transferred in the middle region. The measured curve bears a resemblance with the local minima measured with the first specimen design (Fig. 2). This supports the theory of sensor undulation before the redesign of the specimen. For the further analysis the assumption is made, that the 


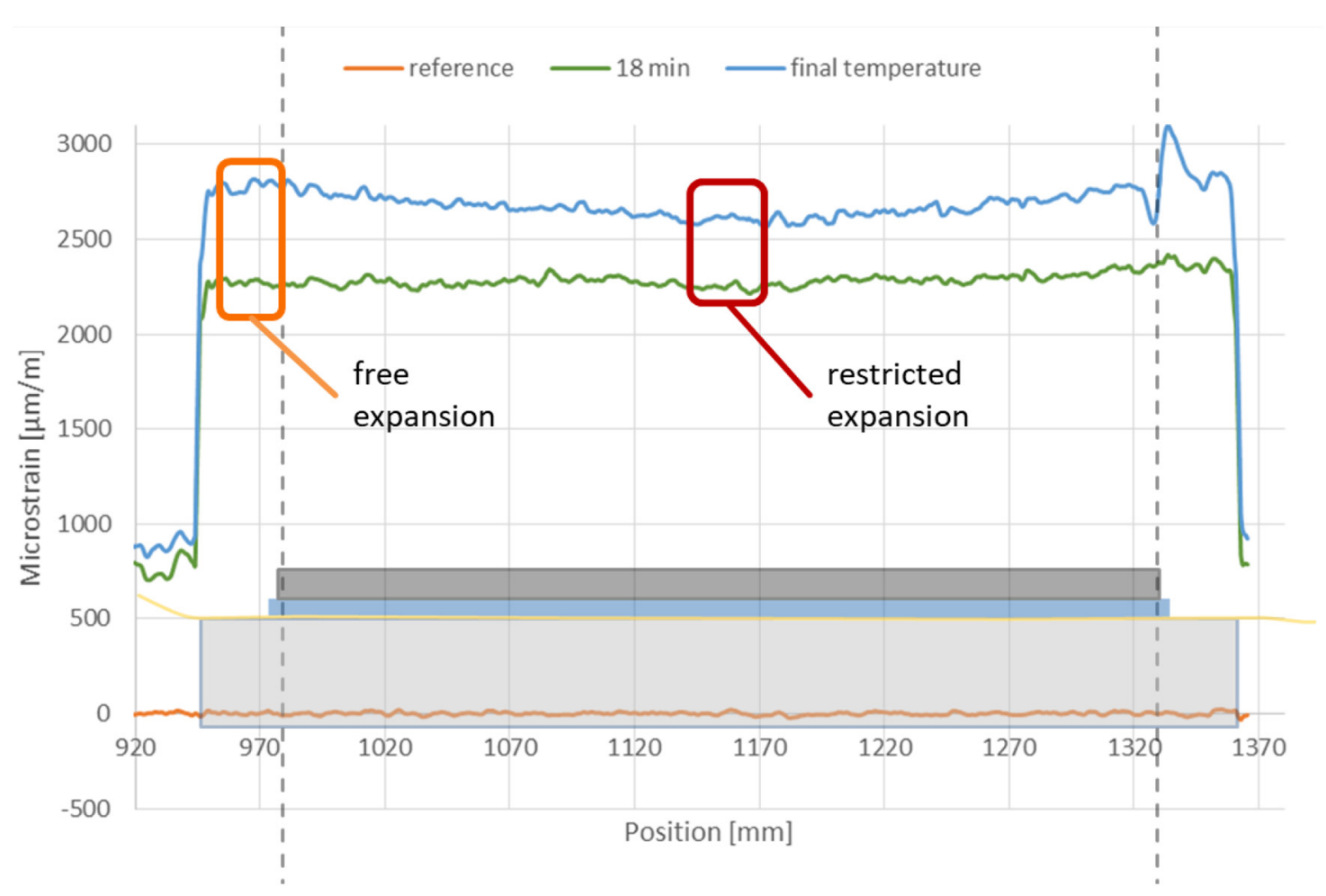

Fig. 7. Deformation during the heating phase.

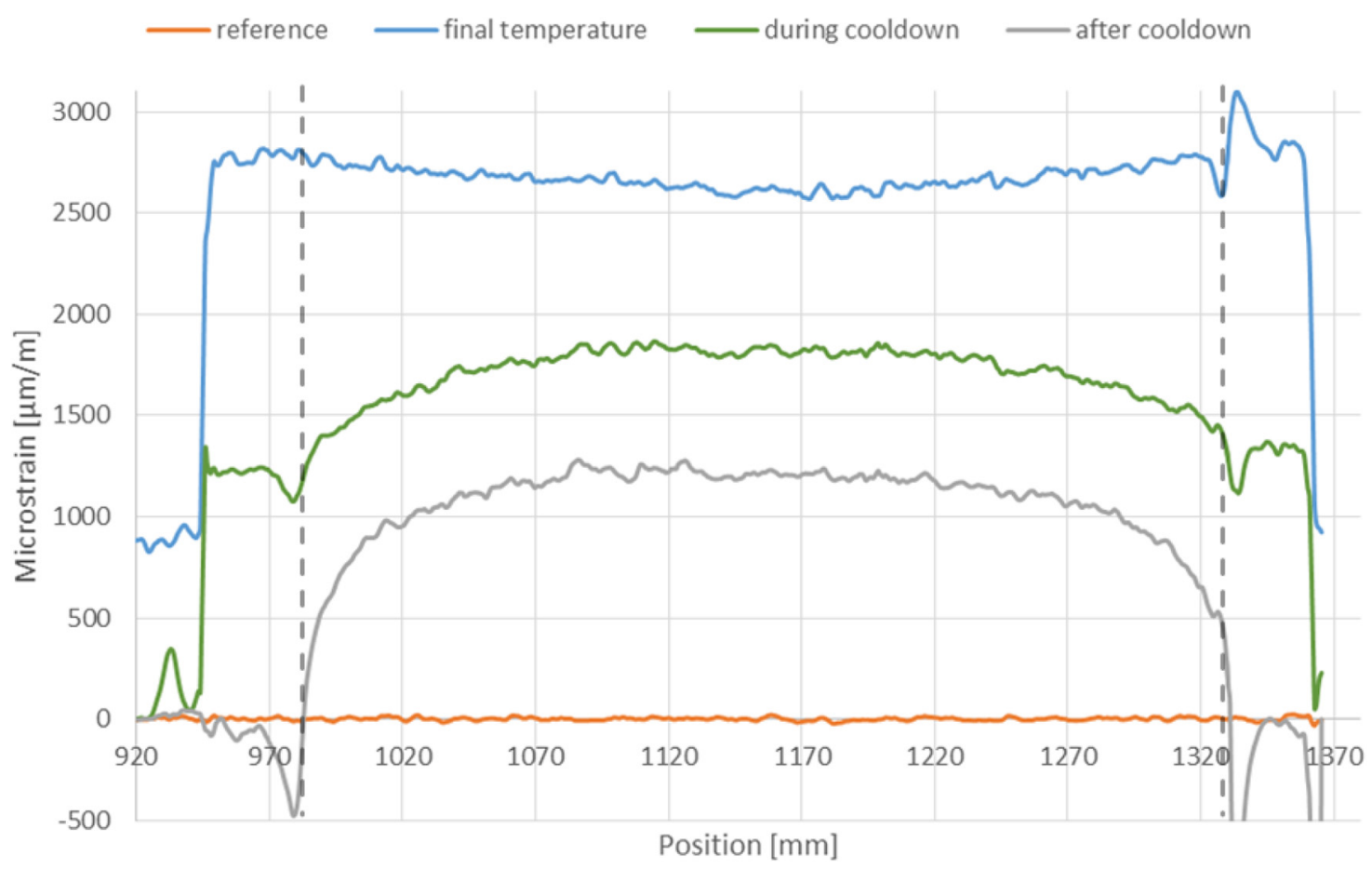

Fig. 8. Deformation during the cooldown.

variations in the deformation are caused by transferred loads, furthermore the deformation gradient indicates the quantity of the load.

The load generated by the residual deformation within the aluminium is counteracted by the load resulting from the compression of the CFRP and the adhesive is transferring the load between both adherents. As expected, the resulting stress is increasing towards the edges of the CFRP plate, the continuous measurement also reveals that the biggest amount is transferred within the first 


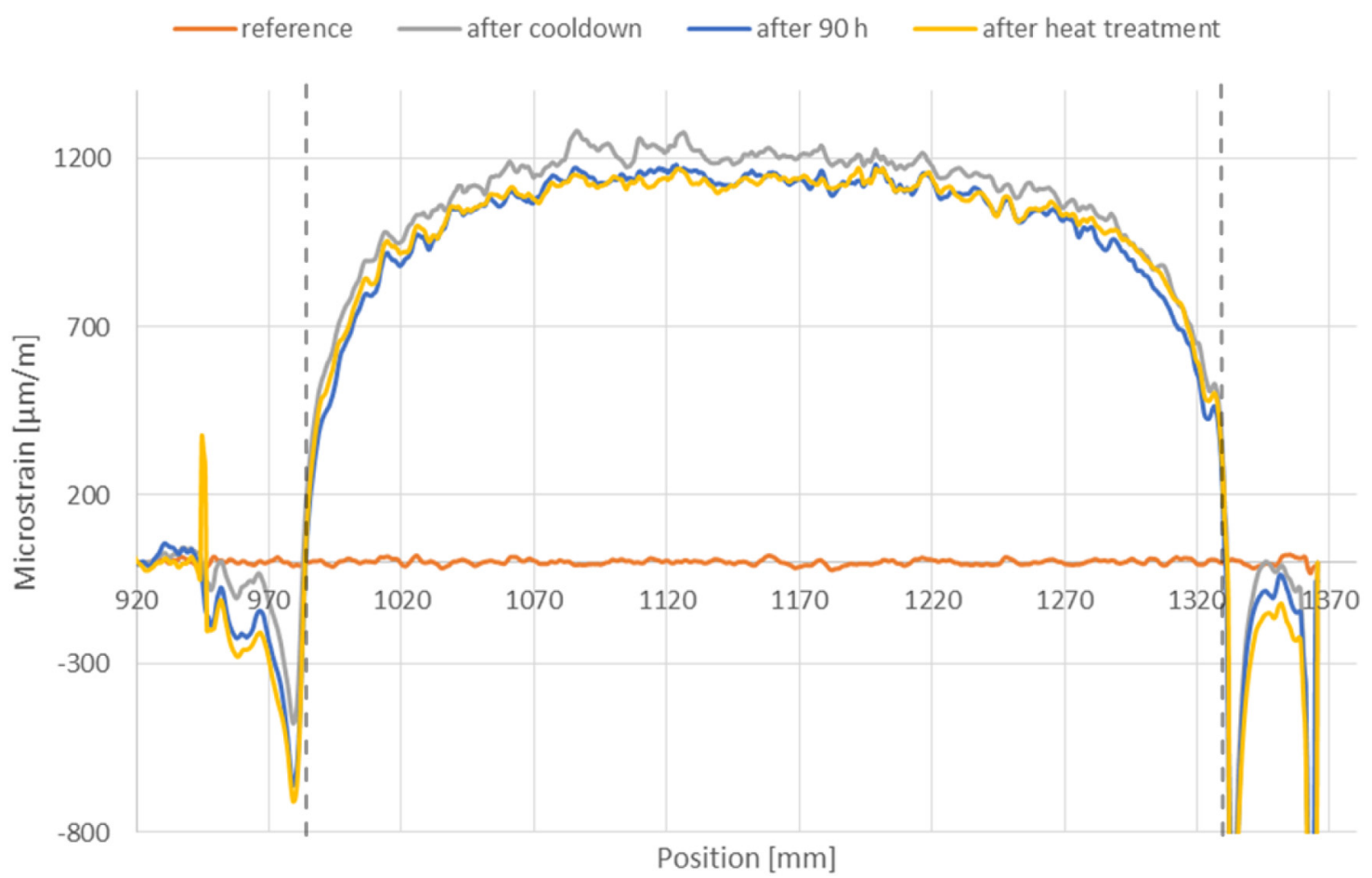

Fig. 9. Deformation after $90 \mathrm{~h}$ rest and heat treatment.

centimetres of the joint. The area in the centre, nearly half of the overall joint length, has only little variance in the displacement hence no significant load transfer.

After a rest for $90 \mathrm{~h}$ with ambient conditions of $23^{\circ} \mathrm{C}$ the specimen was measured again. A minor decrease in the overall deformation was detected (Fig. 9). An attempt to reduce the deformation and the stress was made by heating the specimen above the glass transition temperature of the adhesive which was measured at $89^{\circ} \mathrm{C}$ using differential scanning calorimetry. After $2 \mathrm{~h}$ at $100^{\circ} \mathrm{C}$ and the following cool down to ambient conditions no permanent changes could be detected (Fig. 9).

\section{Conclusions}

Measuring thermal deformation using FOS has shown promising results. Embedding the sensor into the aluminium, as shown in the improved specimen design, allowed the continuous measurement of the deformation within the adherent eliminating the chance of blind spots. During the heating phase the gel-point, representing the formation of structural bonds within the adhesive, was observed as restricted deformation. It was also possible to monitor the accumulation of the remaining deformation in the cooling phase. A post-curing at lower temperatures had no effect on the deformation.

The developed experimental design has provided measurements with a very high level of detail. The acquired data is conclusive with the expected deformation and can be considered a reliable image of the joints behaviour. No obstruction caused by the embedded fibre was detected during the experiments.
Following to this work, the findings will be validated in a simulation of the curing process and mechanic behaviour. In further tests a second sensor fibre will be integrated to measure the deformation of the CFRP. With the maximum acquisition frequency of $23 \mathrm{~Hz}$ at the highest resolution it is also possible to measure dynamic processes such as inductive heating or mechanical loading.

\section{References}

1. G. Habenicht, Kleben (Springer, Berlin, Heidelberg, 2009)

2. F. Eichleiter, Fertigungs- und prozessbedingte Eigenschaften von Klebverbindungen im Karosseriebau [Dissertation]. Technischen Universität Braunschweig, Braunschweig, 2012

3. L.F.M. da Silva, P.M.G.P. Moreira, A.L.D. Loureiro, Determination of the strain distribution in adhesive joints using Fiber Bragg Grating (FBG), J. Adhes. Sci. Technol. 28 (2014) 1480-1499

4. S. Jothibasu, Y. Du, S. Anandan, G.S. Dhaliwal, R.E. Gerald, S.E. Watkins et al., Spatially continuous strain monitoring using distributed fiber optic sensors embedded in carbon fiber composites, Opt. Eng. 58 (2019) 1

5. M. Ramakrishnan, G. Rajan, Y. Semenova, G. Farrell, Overview of fiber optic sensor technologies for strain/ temperature sensing applications in composite materials, Sensors (Basel) 16 (2016), https://doi.org/10.3390/ s16010099

6. X. Bao, L. Chen, Recent progress in Brillouin scattering based fiber sensors, Sensors (Basel) 11 (2011) 4152-4187

7. G. Bolognini, A. Hartog, Raman-based fibre sensors: Trends and applications, Opt. Fiber Technol. 19 (2013) 678-688 
8. B. Yan, J. Li, M. Zhang, J. Zhang, L. Qiao, T. Wang, Raman distributed temperature sensor with optical dynamic difference compensation and visual localization technology for tunnel fire detection, Sensors (Basel) 19 (2019) https://doi. org $/ 10.3390 /$ s19102320

9. A.J. Rogers, V.A. Handerek, Frequency-derived distributed optical-fiber sensing: Rayleigh backscatter analysis, Appl. Opt. 31 (1992) 4091-4095

10. M. Froggatt, J. Moore, High-spatial-resolution distributed strain measurement in optical fiber with Rayleigh scatter, Appl. Opt. 37 (1998) 1735-1740

11. X. Bao, L. Chen, Recent progress in distributed fiber optic sensors, Sensors (Basel) 12 (2012) 8601-8639

12. S.T. Kreger, D.K. Gifford, M.E. Froggatt, B.J. Soller, M.S. Wolfe, High resolution distributed strain or temperature measurements in single- and multi-mode fiber using sweptwavelength interferometry, in Optical Fiber Sensors (ThE42, Washington, D.C.: OSA, 2006)

13. D. Samiec, Distributed fibre-optic temperature and strain measurement with extremely high spatial resolution, Phot. Int. (2012) 10-13

14. K. Yuksel, M. Wuilpart, V. Moeyaert, P. Megret, Optical frequency domain reflectometry: a review, in 11th International Conference on Transparent Optical Networks, edited by M. Jaworski, ICTON '09; June 28, 2009-July 2, 2009 (IEEE; Ponta Delgada, Island of São Miguel, Azores, Portugal. Piscataway, NJ, 2009), pp. 1-5
15. N.A.A. Rahim, M.A. Thoreson, T. Gorney, N. Garg, D.K. Gifford, M.E. Froggatt et al., Superrior fatigue charateristics of fibre optic strain sensors, in Aircraft Structural Integrity Program Conference (ASIP), San Antonio, TX, 2012

16. L. Wong, N. Chowdhury, J. Wang, W.K. Chiu, J. Kodikara, Fatigue damage monitoring of a aomposite step lap joint using distributed optical fibre sensors, Materials (Basel) 9 (2016), https://doi.org/10.3390/ma9050374

17. K. Speck, F. Vogdt, M. Curbach, Y. Petryna, Faseroptische Sensoren zur kontinuierlichen Dehnungsmessung im Beton, Beton- und Stahlbetonbau 114 (2019) 160-167

18. O. Fischer, S. Thoma, S. Crepaz, Quasikontinuierliche faseroptische Dehnungsmessung zur Rissdetektion in Betonkonstruktionen, Beton- und Stahlbetonbau 114 (2019) 150-159

19. Z. Chen, H. Grefe, T. Leusmann, K. Dilger, D. Lowke, Quasicontinous strain measurement with distributed fibre-optic sensors in reinforcement bars and externally bonded CFRP strips at concrete slabs - experiment and modelling. FRPCS-14. Belfast, 2019

20. R.G. Dietrich, Analyse der Wärmeausdehnungs-Inkompatibilität bei Klebverbindungen aus CFK, Stahl und Aluminium bei der Fertigung einer lackierten Karosserie [doctoral thesis]. Technischen Universität München, München, 2017

Cite this article as: Hinrich Grefe, Dennis Weiser, Maja Wanda Kandula, Klaus Dilger, Deformation measurement within adhesive bonds of aluminium and CFRP using advanced fibre optic sensors, Manufacturing Rev. 7, 14 (2020) 